\section{EPV140/\#62 SURVIVAL OUTCOMES IN ENDOMETRIAL CANCER PATIENTS HAVING LYMPHADENECTOMY, SENTINEL NODE MAPPING PLUS BACK-UP LYMPHADENECTOMY AND SENTINEL NODE MAPPING ALONE}

${ }^{1} \mathrm{G}$ Bogani ${ }^{*},{ }^{2} \mathrm{~F}$ Ghezzi, ${ }^{3} \mathrm{R}$ Angioli, ${ }^{4} \mathrm{~A}$ Papadia, ${ }^{5} \mathrm{~A}$ Buda, ${ }^{6} \mathrm{~V}$ Di Donato, ${ }^{3} \mathrm{~F}$ Plotti, ${ }^{7} \mathrm{P}$ De laco, ${ }^{7} \mathrm{AM}$ Perrone, ${ }^{8} \mathrm{~S}$ Ferrero, ${ }^{1} \mathrm{U}$ Leone Roberti Maggiore, ${ }^{4} \mathrm{ML}$ Gasparri, ${ }^{2} \mathrm{~J}$ Casarin, ${ }^{6} \mathrm{~L}$ Muzii, ${ }^{9} \mathrm{M}$ Mueller, ${ }^{10} \mathrm{M}$ Malzoni, ${ }^{5} \mathrm{~F}$ Landoni, ${ }^{6} \mathrm{P}$ Benedetti Panici, ${ }^{1} \mathrm{~F}$ Raspagliesi. ${ }^{1}$ Fondazione IRCCS Istituto Nazionale dei Tumori di Milano, Gynecologic Oncology, Milano, Italy; ' University of Insubria, Gynecologic Oncology, Varese, Italy; ${ }^{3}$ Campus Biomedico, Gynecologic Oncology, Rome, Italy; ${ }^{4}$ University of Lugano, Gynecologic Oncology, Lugano, Switzerland; ${ }^{5}$ Bicocca University, Gynecologic Oncology, Milan, Italy; ${ }^{6}$ Sapienza University, Gynecologic Oncology, Rome, Italy; ' University of Bologna, Gynecologic Oncology, Bologna, Italy; ${ }^{8}$ University of Genoa, Gynecologic Oncology, Genova, Italy; ${ }^{9}$ University of Bern, Gynecologic Oncology, Bern, Switzerland; ${ }^{10}$ Malzoni Medical Center, Gynecologic Oncology, Avellino, Italy

\subsection{6/ijgc-2021-IGCS.210}

Objectives Sentinel node mapping (SNM) has replaced lymphadenectomy for staging surgery in apparent early-stage endometrial cancer (EC). Here, we evaluate the long-term survival of three different approaches of nodal assessment in low, intermediate, and high-risk EC.

Methods This is a multi-institutional retrospective study evaluating long-term outcomes (at least 3 years of follow-up) of EC patients having nodal assessment between 2006 and 2016. In order to reduce possible confounding factors, we applied a propensity-matched algorithm.

Results Charts of 940 patients were evaluated: 174 (18.5\%), 187 (19.9\%), and 579 (61.6\%) having SNM, SNM followed by backup lymphadenectomy and lymphadenectomy, respectively. Applying a propensity score matching algorithm (1:1:2) we selected 500 patients: 125 SNM vs. 125 SNM plus backup lymphadenectomy vs. 250 lymphadenectomy. Baseline characteristics of the study population were similar between groups. The prevalence of nodal disease was $14 \%, 16 \%$, and $12 \%$ in patients having SNM, SNM followed by backup lymphadenectomy and lymphadenectomy, respectively. Overall, 19 (7.6\%) patients were diagnosed with low volume nodal disease ( 7 and 12 patients with micrometastasis and isolated tumor cells). The mean (SD) follow-up time was $62( \pm 11)$ months. The survival analysis comparing the three techniques did not show statistical differences in terms of disease-free $(p=0.750)$ and overall survival $(p=0.899)$. Similarly, the type of nodal assessment did not impact survival outcomes after stratification on the basis of uterine risk factors.

Conclusions SNM provides similar long-term oncologic outcomes than lymphadenectomy. Further evidence is warranted to assess the prognostic value of low-volume disease detected by ultrastaging and the role of molecular/genomic profiling

\section{EPV141/\#627 TOTAL HYSTERECTOMY FOR UNEXPECTED UTERINE LEIOMYOSARCOMA: IMPACT OF SURGERY ON ONCOLOGICAL OUTCOMES}

${ }^{1}$ A Lembo*, ${ }^{2} \mathrm{M}$ Longo, ${ }^{1} \mathrm{E}$ Ervas, ${ }^{1} \mathrm{~V}$ Artuso, ${ }^{1} \mathrm{E}$ Galati, ${ }^{1} \mathrm{~F}$ Ghezzi, ${ }^{3} \mathrm{~J}$ Casarin. ${ }^{1}$ Women's and children hospital F. Del Ponte Hospital - University of Insubria, Obstetrics and Gynecology, Varese, Italy; ${ }^{2}$ F. Del Ponte Hospital - University of Insubria, Obstetrics and Gynecology, Varese, Italy; ${ }^{3} F$. Del Ponte Hospital, University of Insubria, Department of Gynecology and Obstetrics, Varese, Italy

10.1136/ijgc-2021-IGCS.211
Objectives To evaluate the impact of preoperative diagnosis of malignancy on survival in patients surgically treated for apparent early-stage uterine leiomyosarcoma (ULMS).

Methods Data of consecutive patients who underwent total hysterectomy at Del Ponte Hospital, (Varese-Italy) between January 2000 and December 2019 were retrieved. Only cases with histologically proven ULMS at final diagnosis were included and stratified according with the preoperative finding of malignancy into: 'Suspicious ULMS' vs. 'unexpected ULMS'. Demographic, pathologic and surgical-related characteristics were compared. Survivals curves were estimated with Kaplan-Meier methods and predictors of recurrence were investigated.

Results Overall 36 patients ULMS were included, 24 and 12 'unexpected ULMS' and 'suspicious ULMS', respectively. No significant differences between the groups in terms of baseline characteristics and surgical approach (minimally-invasive approach: 3, 25\% vs. 15, 62.5\%, p=0.08) were found. The morcellation of the uterus was less likely performed in patients in 'suspicious ULMS' (18, 33\% vs.14, 58.33\%; $\mathrm{p}=0.005)$. The survival analysis did not show statistical differences between the groups. No differences in survival (DFS $(\log$-rank $=0.28)$ and OS (log-rank=0.78).

Details on recurrence are reported (table 1). No predictors of relapse were found, including uterine morcellation $(41.67 \%$ vs. $66.67 \%, \mathrm{p}=0.15)$.

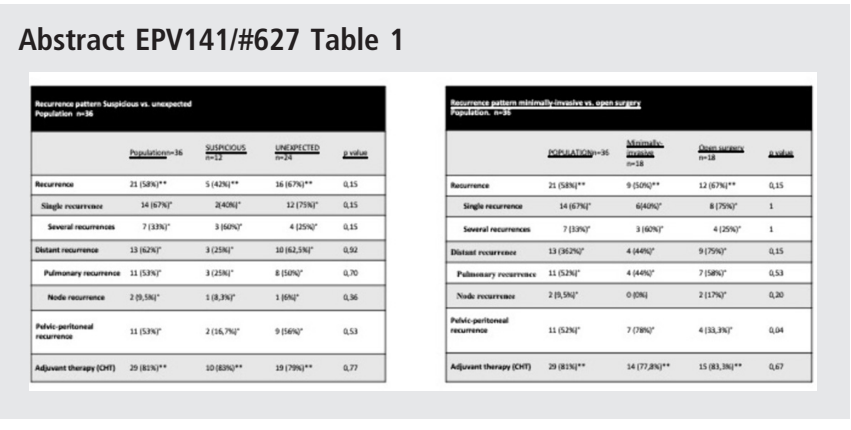

Conclusions Patients undergoing hysterectomy for ULMS have poor prognosis regardless the surgical approach. In our population, preoperative suspicious of malignancy did not influence survival outcomes and morcellation did not seem to have a detrimental effect on recurrence rate.

Larger studies are warranted to confirm our findings.

\section{EPV142/\#638 DEMONSTRATION OF A LEARNING CURVE IN THE INITIATION OF SENTINEL LYMPH NODE MAPPING IN ENDOMETRIAL CANCER IN A WELSH TERTIARY GYNAE CANCER CENTRE}

L Rider*, K Lim, R Howells, A Sharma, J Oliviera Davies, S Jones. University Hospital of Wales, Gynae Oncology, Cardiff, UK

\subsection{6/ijgc-2021-IGCS.212}

Objectives To audit the outcomes of sentinel lymph node mapping in a Welsh cancer centre, in order to demonstrate a sufficient learning curve to adopt sentinel lymph node biopsy as the mainstay of surgical lymph node mapping in endometrial cancer. 
Methods There are no current standards against which to audit the departments learning curve for adoption of sentinel lymph node mapping as endometrial cancer staging. We identified published quality indicators for sentinel lymph node mapping - including $<5 \%$ false negative rate, $>20$ cases per surgeon performing the procedure, successful bilateral mapping in $>50 \%$ of cases. Our local gynae oncology database was searched to identify all cases of sentinel lymph node dissection for endometrial and cervical cancer. Data from the gynae oncology database and the patients electronic clinical record was then collated and analysed using excel.

Results 43 patients were identified having undergone a sentinel lymph node biopsy \pm lymphadenectomy for endometrial or cervical cancer. Bilateral sentinel lymph nodes were mapped in $67.4 \%$ of cases. In the first $21 / 43$ patients $57.1 \%$ were mapped, comparative to $77.3 \%$ in latter $22 / 43$ patients. 38 sides with successful lymph node mapping and lymphadenectomy were identified. Sentinel lymph nodes had a 33\% sensitivity for identifying lymph node metastasis in the first half of the data set comparative to $100 \%$ in the latter half.

Conclusions The data demonstrated a significant learning curve, within the department, in the successful mapping of sentinel lymph nodes in endometrial cancer.

\section{EPV143/\#656 RETROSPECTIVE DATA ANALYSIS OF HOSPITAL SANTA MARCELINA, SAO PAULO-SP, BRAZIL}

M Mesquita*, M Brandão, C Sousa, J Barbosa, S Sanches, I Manchini, C Gomez, M Silva, T Almeida. Casa de Saúde Santa Marcelina, Gynecologic Oncology, Sao Paulo, Brazil

\subsection{6/ijgc-2021-IGCS.213}

Objectives Evaluate the epidemiological aspects of patients with endometrial cancer, based on statistics from the Oncology Gynecology Center of Santa Marcelina Hospital in Sao Paulo, Brazil between 2011 to 2018 .

Methods Evaluate the epidemiological aspects of patients with endometrial cancer (EC), based on statistics from the Oncology Gynecology Center of Santa Marcelina Hospital in Sao Paulo, Brazil between 2011 to 2018 .

Results The median age at diagnosis was 63 years and the diagnosed cases were predominantly white ethnicity (51\%). Bleeding after menopause was the most frequent symptom reported (77.8\%). Among the cases analyzed, 36 nulliparous patients presented endometrial cancer (15\%). The most prevalent histological type was endometrioid adenocarcinoma $(66.1 \%)$. The most frequent tumor staging was IA with $30.9 \%$, followed by IB $18.83 \%$, II $2 \%$, IIIA $8 \%$, IIIB $9.2 \%$ IIIC1 $4.6 \%$, IIIC2 $6.69 \%$, IVA $0.42 \%$ and IVB $16.74 \%$. Surgical staging with hysterectomy and bilateral adnexectomy represented $76.9 \%$ and the most frequent adjuvant treatment was brachytherapy (53.1\%). Seventy patients underwent brachytherapy and pelvic radiotherapy (29.9\%) and 38 patients underwent adjuvant brachytherapy, radiotherapy and chemotherapy as an adjuvant (15.9\%). An overall survival rate of $65 \%$ and a mortality rate of $29 \%$ over the 5 -year period have been identified.

Conclusions EC is the eighth most frequent gynecological tumor in Brazil. Data analysis allowed to corroborate the most common clinical symptom and the frequent histological type in the literature. This neoplasm classically presents early symptoms and curative treatment, however the data analysis shows a high death rate and diagnosis of advanced disease.
So, the endometrioid type, doesn't have the best prognosis always and needs a better molecular analysis to optimize therapy,to reduce mortality.

\section{EPV144/\#76 THE TUNISIAN COUNTRY-SPECIFIC GUIDELINES FOR ENDOMETRIAL CANCER}

${ }^{1} \mathrm{M}$ Ghalleb*, 'L Naija, ${ }^{1} \mathrm{~A}$ Chabchoub, ${ }^{1} \mathrm{I}$ Zemni, ${ }^{2} \mathrm{~S}$ Zaraa, ${ }^{2} \mathrm{~S}$ Yahyaoui, ${ }^{3} \mathrm{~F}$ Mghirbi, ${ }^{3} \mathrm{M}$ Ayadi, ${ }^{3} \mathrm{~N}$ Chraiet, ${ }^{1} \mathrm{M}$ Slimane, ${ }^{4} \mathrm{~L}$ Charfi, ${ }^{4} \mathrm{~K}$ Mrad, ${ }^{2} \mathrm{C}$ Nasr, ${ }^{3} \mathrm{~A}$ Mezlini, ${ }^{1} \mathrm{M}$ Hechiche, ${ }^{1} \mathrm{~K}$ Rahal. 'Salah Azaiez Institute of Oncology, Surgical Oncology, Tunis, Tunisia; ${ }^{2}$ Salah Azaiez Institute of Oncology, Radiation Oncology, Tunis, Tunisia; ${ }^{3}$ Salah Azaiez Institute of Oncology, Medical Oncology, Tunis, Tunisia; ${ }^{4}$ Salah Azaiez Institute of Oncology, Pathology Deaprtment, Tunis, Tunisia

\subsection{6/ijgc-2021-IGCS.214}

Objectives Endometrial cancer is the second gynecologic cancer. The varying tumors profile from country to country and the difference in the means available in each country have raised the need for a country-specific guideline. We aim to Present the Tunisian guideline for endometrial cancer

Methods All relevant international and national scientific literature available from 2016 to 2021 was used to establish this guideline.

Results This guideline was made by the Gynecologic Oncology Multidisciplinary team of the National Cancer center. Three questions were asked. What is the actual state of the art? Could it be applied in our country? If not, can we adapt the guideline to our reality?. During the consensus, the panel tried to cope between the actual state of the art and the Tunisian Field reality. The main limitations were the Distant radiation appointment, the patient loss to follow up, and the non-systematic use of biological markers. The 2009 FIGO classification was used to stage our patients. For stage I disease, The ESMO 2016 risk classification was used. One preoperatory and composed of three levels of risks low, intermediate, and high risk. The other classification is post-operatory and comprises low, Intermediate, high-intermediate, and high-risk levels. Based on this Data and our country reality panel developed recommendations.

Conclusions A country-specific guideline based on the international state of the art is more effective to offer the best quality of care available to our patients. It would also point to the lack and what needs to be done to keep on improving the health system.

\section{EPV145/\#82 MULTICENTRIC PREDICTIVE SCORE VALIDATION FOR NODAL ASSESSMENT IN ENDOMETRIAL CANCER PATIENTS: PRELIMINARY DATA}

${ }^{1}$ VA Capozzi, ${ }^{2} \mathrm{G}$ Sozzi, ${ }^{3} \mathrm{~A}$ Rosati ${ }^{*},{ }^{3} \mathrm{~S}$ Restaino, ${ }^{1} \mathrm{G}$ Gambino, ${ }^{1} \mathrm{~A}$ Cianciolo, ${ }^{4} \mathrm{M}$ Ceccaroni, ${ }^{2} \mathrm{~V}$ Chiantera, ${ }^{5} \mathrm{~S}$ Uccella, ${ }^{5} \mathrm{M}$ Franchi, ${ }^{3} \mathrm{G}$ Scambia, ${ }^{3} \mathrm{~F}$ Fanfani, ${ }^{1} \mathrm{R}$ Berretta. ${ }^{1}$ University of Parma, Department of Medicine and Surgery, Parma, Italy; ${ }^{2}$ University of Palermo, Department of Gynecologic Oncology, Arnas Civico Di Cristina Benfratelli, Palermo, Italy; ${ }^{3}$ Università Cattolica del Sacro Cuore, Department of Woman and Child Health and Public Health, Woman Health Area, Fondazione Policlinico Universitario A. Gemelli Irccs, Roma, Italy; ${ }^{4}$ RCCS Sacro Cuore Don Calabria Hospital, Gynecologic Oncology, Minimally-Invasive Pelvic Surgery, International School of Surgical Anatomy, Negrar, Italy; ${ }^{5}$ University of Verona, Department of Obstetrics and Gynecology, Verona, Italy

\subsection{6/ijgc-2021-IGCS.215}

Objectives Sentinel lymph node (SLN) is considered the standard of care in early-stage endometrial cancer (EC) patients. In case of SLN failure, a side-specific lymphadenectomy of the 